\title{
Article \\ Applications of the Fractional Calculus in Fuzzy Differential Subordinations and Superordinations
}

\author{
Alina Alb Lupaş ${ }^{D}$ \\ Department of Mathematics and Computer Science, University of Oradea, 1 Universitatii Street, \\ 410087 Oradea, Romania; dalb@uoradea.ro or alblupas@gmail.com
}

check for updates

Citation: Alb Lupaş, A. Applications of the Fractional Calculus in Fuzzy Differential Subordinations and Superordinations. Mathematics 2021, 9, 2601. https://doi.org/10.3390/ math9202601

Academic Editors: Sorin Nadaban and Ioan Dzitac

Received: 17 September 2021

Accepted: 14 October 2021

Published: 15 October 2021

Publisher's Note: MDPI stays neutral with regard to jurisdictional claims in published maps and institutional affiliations.

Copyright: (C) 2021 by the author. Licensee MDPI, Basel, Switzerland. This article is an open access article distributed under the terms and conditions of the Creative Commons Attribution (CC BY) license (https:// creativecommons.org/licenses/by/ $4.0 /)$.

\begin{abstract}
The fractional integral of confluent hypergeometric function is used in this paper for obtaining new applications using concepts from the theory of fuzzy differential subordination and superordination. The aim of the paper is to present new fuzzy differential subordinations and superordinations for which the fuzzy best dominant and fuzzy best subordinant are given, respectively. The original theorems proved in the paper generate interesting corollaries for particular choices of functions acting as fuzzy best dominant and fuzzy best subordinant. Another contribution contained in this paper is the nice sandwich-type theorem combining the results given in two theorems proved here using the two theories of fuzzy differential subordination and fuzzy differential superordination.
\end{abstract}

Keywords: fuzzy differential subordination; fuzzy differential superordination; fuzzy best dominant; fuzzy best subordinant; fractional integral; confluent hypergeometric function

\section{Introduction}

The concept of fuzzy differential subordination and its dual, the concept of fuzzy differential superordination were introduced in the last decade as a result of the trend for adapting the notion of fuzzy set to different topics of research. Even if the notion of fuzzy set didn't look promising when it was first introduced by Lotfi A. Zadeh in his paper published in 1965 [1], in the recent years it became part of many branches of science and scientific research. Mathematical sciences also aimed for introducing and using fuzzifications of the already established classical theories in different fields of research. The review paper published in 2017 [2] shows some parts of the history of the fuzzy set notion and how Zadeh's new concept has revolutionized Soft Computing and Artificial Intelligence as well as other fields of science and technique. Another review paper published as part of a special issue dedicated to celebrating Zadeh's birth centenary [3] shows other aspects from the development process of fuzzy logic based on the notion of fuzzy set.

Fuzzy sets theory was connected to geometric function theory in 2011 when the notion of fuzzy subordination was introduced [4] with as inspiration the theory of differential subordination initiated by Miller and Mocanu in 1978 [5] and 1981 [6]. The core of the theory of differential subordination was gradually adapted to fuzzy sets notions in the subsequent years [7-9] following the main lines of research as found in the monograph published in 2000 [10]. Obtaining fuzzy subordination and superordination results involving operators was a topic approached early in the study of fuzzy subordinations. Fuzzy differential subordinations were first obtained using a convolution product of Sălăgean operator and Ruscheweyh derivative in 2013 [11]. The study following this line of research was continued and, in 2015, fuzzy differential subordinations were investigated using Ruscheweyh operator [12], and then using a multiplier transformation in 2016 [13]. Fuzzy differential subordinations for prestar-like functions of complex order and some applications were published in 2017 [14] and in the same year, the dual notion of fuzzy differential superordination was introduced [15]. After this event, the two notions were used together in many investigations giving applications such as in [16] and adding operators to the research such 
as the Srivastava-Attitya operator in [17]. In recent years, theories of fuzzy differential subordination and fuzzy differential superordination developed nicely. New fuzzy differential subordinations associated with integral operators were investigated in $[18,19]$ and a linear operator was considered for the study in [20]. New applications of Sălăgean and Ruscheweyh operators for obtaining fuzzy differential subordinations were investigated in [21] and fuzzy differential subordinations based upon the Mittag-Leffler-type Borel distribution also emerged [22].

The present paper considers for the investigation from a fuzzy point of view an operator obtained using fractional integrals of confluent hypergeometric function. The investigation of such an operator for obtaining new fuzzy differential subordinations and superordinations results is motivated by recent fuzzy related investigations that considered a fractional integral associated with generalized Mittag-Leffler function [23], a fuzzy Atangana-Baleanu fractional derivative operator in [24] and fuzzy differential subordinations obtained using a hypergeometric integral operator [25].

Fractional calculus has had tremendous development in recent years proving to have applications in many research domains such as physics, engineering, turbulence, electric networks, biological systems with memory, computer graphics, etc. As an example, the Korteweg-de Vries equation, developed to represent a broad spectrum of physics behaviors of the evolution and association of nonlinear waves, is studied using a new integral transform where the fractional derivative is proposed in the Caputo sense [26]. Related to biological systems, examples can be given as the new study on the mathematical modelling of the human liver with the Caputo-Fabrizio fractional derivative proposed in [27] and fractional calculus analysis of the transmission dynamics of the dengue infection seen in [28].

Numerous interesting approaches were taken into account regarding fractional integral calculus and new results have emerged in an impressive number of papers published lately. A study of fractional integral operators involving a certain generalized multi-index Mittag-Leffler function was conducted in [29]. Integral inequalities were investigated using integral operators defined with fractional integral of Gauss hypergeometric function [30] and using $\gamma$-convex functions and a generalized fractional integral operator based on Raina's function [31]. Subclasses of analytic functions defined using a fractional integral operator were introduced and studied in [32,33]. Applications of differential subordination theory to analytic and $p$-valent functions defined by a generalized fractional differintegral operator were presented in [34] and a new fractional integral operator is used in the study on the Mittag-Leffler-confluent hypergeometric function [35].

Following the research line in which fuzzy differential subordinations and superordinations are connected to operators, the interesting fractional integral of confluent hypergeometric function introduced and investigated in [36] using the classical theories of differential subordinations and superordinations is further considered from this new perspective involving fuzzy set theory notions. The purpose of the investigation is to present new fuzzy differential subordinations and superordinations which lead to interesting corollaries when using functions with remarkable geometric properties known from geometric function theory as fuzzy best dominant and fuzzy best subordinant, respectively.

\section{Preliminaries}

The study presented in this paper is done in the general context of geometric function theory.

The unit disc of the complex plane is denoted by $U=\{z \in \mathbb{C}:|z|<1\}$ and the class of analytic functions in $U$ by $\mathcal{H}(U)$. For $n$ a positive integer and $a \in \mathbb{C}, \mathcal{H}[a, n]$ denotes the subclass of $\mathcal{H}(U)$ consisting of functions written in the form $f(z)=a+a_{n} z^{n}+a_{n+1} z^{n+1}+$ $\ldots, z \in U$.

A function with beautiful applications in defining operators is the fractional integral of order $\lambda$ given as: 
Definition 1 ([37]). The fractional integral of order $\lambda(\lambda>0)$ is defined for a function $f$ by

$$
D_{z}^{-\lambda} f(z)=\frac{1}{\Gamma(\lambda)} \int_{0}^{z} \frac{f(t)}{(z-t)^{1-\lambda}} d t
$$

where $f$ is an analytic function in a simply connected region of the z-plane containing the origin, and the multiplicity of $(z-t)^{\lambda-1}$ is removed by requiring $\log (z-t)$ to be real, when $(z-t)>0$.

The definitions of the notions used in the present investigation are next recalled.

Confluent (or Kummer) hypergeometric function is defined as:

Definition 2 ([10] p. 5). Let $a$ and $c$ be complex numbers with $c \neq 0,-1,-2, \ldots$ and consider

$$
\phi(a, c ; z)={ }_{1} F_{1}(a, c ; z)=1+\frac{a}{c} \frac{z}{1 !}+\frac{a(a+1)}{c(c+1)} \frac{z^{2}}{2 !}+\ldots, z \in U .
$$

This function is called confluent (Kummer) hypergeometric function, is analytic in $\mathbb{C}$ and satisfies Kummer's differential equation

$$
z w^{\prime \prime}(z)+(c-z) w^{\prime}(z)-a w(z)=0
$$

The operator introduced in [36] using the fractional integral of confluent hypergeometric function is given in the following definition:

Definition 3 ([36]). Let $a$ and $c$ be complex numbers with $c \neq 0,-1,-2, \ldots$ and $\lambda>0$. We define the fractional integral of confluent hypergeometric function

$$
\begin{gathered}
D_{z}^{-\lambda} \phi(a, c ; z)=\frac{1}{\Gamma(\lambda)} \int_{0}^{z} \frac{\phi(a, c ; t)}{(z-t)^{1-\lambda}} d t= \\
\frac{1}{\Gamma(\lambda)} \frac{\Gamma(c)}{\Gamma(a)} \sum_{k=0}^{\infty} \frac{\Gamma(a+k)}{\Gamma(c+k) \Gamma(k+1)} \int_{0}^{z} \frac{t^{k}}{(z-t)^{1-\lambda}} d t .
\end{gathered}
$$

Remark 1 ([36]). The fractional integral of confluent hypergeometric function can be written

$$
D_{z}^{-\lambda} \phi(a, c ; z)=\frac{\Gamma(c)}{\Gamma(a)} \sum_{k=0}^{\infty} \frac{\Gamma(a+k)}{\Gamma(c+k) \Gamma(\lambda+k+1)} z^{k+\lambda},
$$

after a simple calculation. Evidently $D_{z}^{-\lambda} \phi(a, c ; z) \in \mathcal{H}[0, \lambda]$.

For the concept of fuzzy differential subordination to be used, the following notions are necessary:

Definition 4 ([38]). A pair $\left(A, F_{A}\right)$, where $F_{A}: X \rightarrow[0,1]$ and $A=\left\{x \in X: 0<F_{A}(x) \leq 1\right\}$ is called the fuzzy subset of $X$. The set $A$ is called the support of the fuzzy set $\left(A, F_{A}\right)$ and $\bar{F}_{A}$ is called the membership function of the fuzzy set $\left(A, F_{A}\right)$. One can also denote $A=\operatorname{supp}\left(A, F_{A}\right)$.

Remark 2 ([38]). If $A \subset X$, then $F_{A}(x)=\left\{\begin{array}{l}1, \text { if } x \in A \\ 0, \text { if } x \notin A\end{array}\right.$.

For a fuzzy subset, the real number 0 represents the smallest membership degree of a certain $x \in X$ to $A$ and the real number 1 represents the biggest membership degree of a certain $x \in X$ to A.

The empty set $\varnothing \subset X$ is characterized by $F_{\varnothing}(x)=0, x \in X$, and the total set $X$ is characterized by $F_{X}(x)=1, x \in X$. 
Definition 5 ([4]). Let $D \subset \mathbb{C}, z_{0} \in D$ be a fixed point and let the functions $f, g \in \mathcal{H}(D)$. The function $f$ is said to be fuzzy subordinate to $g$ and write $f \prec_{\mathcal{F}} g$ or $f(z) \prec_{\mathcal{F}} g(z)$, if are satisfied the conditions:

(1) $f\left(z_{0}\right)=g\left(z_{0}\right)$,

(2) $F_{f(D)} f(z) \leq F_{g(D)} g(z), z \in D$.

Definition 6 ([8] Definition 2.2). Let $\psi: \mathbb{C}^{3} \times U \rightarrow \mathbb{C}$ and h univalent in $U$, with $\psi(a, 0 ; 0)=$ $h(0)=a$. If $p$ is analytic in $U$, with $p(0)=a$ and satisfies the (second-order) fuzzy differential subordination

$$
F_{\psi\left(\mathbb{C}^{3} \times U\right)} \psi\left(p(z), z p^{\prime}(z), z^{2} p^{\prime \prime}(z) ; z\right) \leq F_{h(U)} h(z), \quad z \in U,
$$

then $p$ is called a fuzzy solution of the fuzzy differential subordination. The univalent function $q$ is called a fuzzy dominant of the fuzzy solutions of the fuzzy differential subordination, or more simply a fuzzy dominant, if $F_{p(U)} p(z) \leq F_{q(U)} q(z), z \in U$, for all $p$ satisfying (4). A fuzzy dominant $\widetilde{q}$ that satisfies $F_{\widetilde{q}(U)} \tilde{q}(z) \leq F_{q(U)} q(z), z \in U$, for all fuzzy dominants $q$ of (4) is said to be the fuzzy best dominant of (4).

Definition 7 ([11]). Let $\varphi: \mathbb{C}^{3} \times U \rightarrow \mathbb{C}$ and let $h$ be analytic in $U$. If $p$ and $\varphi\left(p(z), z p^{\prime}(z)\right.$, $\left.z^{2} p^{\prime \prime}(z) ; z\right)$ are univalent in $U$ and satisfy the (second-order) fuzzy differential superordination

$$
F_{h(U)} h(z) \leq F_{\varphi\left(\mathbb{C}^{3} \times U\right)} \varphi\left(p(z), z p^{\prime}(z), z^{2} p^{\prime \prime}(z) ; z\right), \quad z \in U,
$$

i.e.,

$$
h(z) \prec \mathcal{F} \varphi\left(p(z), z p^{\prime}(z), z^{2} p^{\prime \prime}(z) ; z\right), \quad z \in U,
$$

then $p$ is called a fuzzy solution of the fuzzy differential superordination. An analytic function $q$ is called fuzzy subordinant of the fuzzy differential superordination, or more simply a fuzzy subordination if

$$
F_{q(U)} q(z) \leq F_{p(U)} p(z), z \in U,
$$

for all $p$ satisfying (5). A univalent fuzzy subordination $\widetilde{q}$ that satisfies $F_{q(U)} q \leq F_{q(U)} \widetilde{q}$ for all fuzzy subordinate $q$ of (5) is said to be the fuzzy best subordinate of (5). Please note that the fuzzy best subordinant is unique to a relation of $U$.

The purpose of this paper is to obtain several fuzzy differential subordination and superordination results, using the following known results.

Definition 8 ([8]). Denote by $Q$ the set of all functions $f$ that are analytic and injective on $\bar{U} \backslash E(f)$, where $E(f)=\left\{\zeta \in \partial U: \lim _{z \rightarrow \zeta} f(z)=\infty\right\}$, and are such that $f^{\prime}(\zeta) \neq 0$ for $\zeta \in \partial U \backslash E(f)$.

Lemma 1 ([8]). Let the function $q$ be univalent in the unit disc $U$ and $\theta$ and $\phi$ be analytic in $a$ domain $D$ containing $q(U)$ with $\phi(w) \neq 0$ when $w \in q(U)$. Set $Q(z)=z q^{\prime}(z) \phi(q(z))$ and $h(z)=\theta(q(z))+Q(z)$. Suppose that

1. $Q$ is starlike univalent in $U$ and

2. $\operatorname{Re}\left(\frac{z h^{\prime}(z)}{Q(z)}\right)>0$ for $z \in U$.

If $p$ is analytic with $p(0)=q(0), p(U) \subseteq D$ and

$$
F_{p(U)} \theta(p(z))+z p^{\prime}(z) \phi(p(z)) \leq F_{h(U)} \theta(q(z))+z q^{\prime}(z) \phi(q(z)),
$$

then

$$
F_{p(U)} p(z) \leq F_{q(U)} q(z)
$$

and $q$ is the fuzzy best dominant. 
Lemma 2 ([11]). Let the function $q$ be convex univalent in the open unit disc $U$ and $v$ and $\phi$ be analytic in a domain $D$ containing $q(U)$. Suppose that

1. $\operatorname{Re}\left(\frac{v^{\prime}(q(z))}{\phi(q(z))}\right)>0$ for $z \in U$ and

2. $\psi(z)=z q^{\prime}(z) \phi(q(z))$ is starlike univalent in $U$. and

If $p(z) \in \mathcal{H}[q(0), 1] \cap Q$, with $p(U) \subseteq D$ and $v(p(z))+z p^{\prime}(z) \phi(p(z))$ is univalent in $U$

$$
F_{q(U)} v(q(z))+z q^{\prime}(z) \phi(q(z)) \leq F_{p(U)} v(p(z))+z p^{\prime}(z) \phi(p(z))
$$

then

$$
F_{q(U)} q(z) \leq F_{p(U)} p(z)
$$

and $q$ is the fuzzy best subordinant.

\section{Main Results}

The first fuzzy subordination result obtained using the operator given by (2) is the following theorem:

Theorem 1. Let the function $q$ be analytic and univalent in $U$ such that $q(z) \neq 0$ and $\left(\frac{D_{z}^{-\lambda} \phi(a, c ; z)}{z}\right)^{\delta}$ $\in \mathcal{H}(U)$, for all $z \in U$, where $a, c$ be complex numbers with $c \neq 0,-1,-2, \ldots$ and $\lambda, \delta>0$. Suppose that $\frac{z q^{\prime}(z)}{q(z)}$ is starlike univalent in $U$. Let

$$
\operatorname{Re}\left(\frac{z q^{\prime \prime}(z)}{q^{\prime}(z)}-\frac{z q^{\prime}(z)}{q(z)}+\frac{2 \mu}{\beta}(q(z))^{2}+\frac{\xi}{\beta} q(z)+1\right)>0,
$$

for $\alpha, \xi, \mu, \beta \in \mathbb{C}, \beta \neq 0, z \in U$ and

$$
\begin{gathered}
\psi_{\lambda}^{a, c}(\delta, \alpha, \xi, \mu, \beta ; z):=\alpha+\beta \delta\left[\frac{z\left(D_{z}^{-\lambda} \phi(a, c ; z)\right)^{\prime}}{D_{z}^{-\lambda} \phi(a, c ; z)}-1\right]+ \\
\mu\left[\frac{D_{z}^{-\lambda} \phi(a, c ; z)}{z}\right]^{2 \delta}+\xi\left[\frac{D_{z}^{-\lambda} \phi(a, c ; z)}{z}\right]^{\delta} .
\end{gathered}
$$

If $q$ satisfies the following fuzzy subordination

$$
F_{\psi_{\lambda}^{a, c}(U)} \psi_{\lambda}^{a, c}(\delta, \alpha, \xi, \mu, \beta ; z) \leq F_{q(U)}\left(\alpha+\beta \frac{z q^{\prime}(z)}{q(z)}+\mu(q(z))^{2}+\xi q(z)\right),
$$

for $\alpha, \xi, \mu, \beta \in \mathbb{C}, \beta \neq 0$, then

$$
F_{D_{z}^{-\lambda} \phi(U)}\left(\frac{D_{z}^{-\lambda} \phi(a, c ; z)}{z}\right)^{\delta} \leq F_{q(U)} q(z), \quad z \in U,
$$

and $q$ is the best dominant.

Proof. Define $p(z):=\left(\frac{D_{z}^{-\lambda} \phi(a, c ; z)}{z}\right)^{\delta}, z \in U, z \neq 0$. Differentiating it we obtain $p^{\prime}(z)=$ $-\frac{\delta}{z} p(z)+\delta\left(\frac{D_{z}^{-\lambda} \phi(a, c ; z)}{z}\right)^{\delta-1} \frac{\left(D_{z}^{-\lambda} \phi(a, c ; z)\right)^{\prime}}{z}$. Then $\frac{z p^{\prime}(z)}{p(z)}=\delta\left[\frac{z\left(D_{z}^{-\lambda} \phi(a, c ; z)\right)^{\prime}}{D_{z}^{-\lambda} \phi(a, c ; z)}-1\right]$.

By setting $\theta(w):=\mu w^{2}+\xi w+\alpha$ and $Q(w):=\frac{\beta}{w}$, it is evident that $\theta$ is analytic in $\mathbb{C}$, $\phi$ is analytic in $\mathbb{C} \backslash\{0\}$ and $\phi(w) \neq 0, w \in \mathbb{C} \backslash\{0\}$.

Considering $Q(z)=z q^{\prime}(z) \phi(q(z))=\beta \frac{z q^{\prime}(z)}{q(z)}$ and $h(z)=Q(z)+\theta(q(z))=\alpha+$ $\beta \frac{z q^{\prime}(z)}{q(z)}+\mu(q(z))^{2}+\xi q(z)$, we deduce that $Q$ is starlike univalent in $U$. 
Differentiating we obtain $h^{\prime}(z)=\xi+q^{\prime}(z)+2 \mu q(z) q^{\prime}(z)+\beta \frac{\left(q^{\prime}(z)+z q^{\prime \prime}(z)\right) q(z)-z\left(q^{\prime}(z)\right)^{2}}{(q(z))^{2}}$ and $\frac{z h^{\prime}(z)}{Q(z)}=\frac{z h^{\prime}(z)}{\beta \frac{z q^{\prime}(z)}{q(z)}}=1+\frac{\xi}{\beta} q(z)+\frac{2 \mu}{\beta}(q(z))^{2}-\frac{z q^{\prime}(z)}{q(z)}+\frac{z q^{\prime \prime}(z)}{q^{\prime}(z)}$, which imply that $\operatorname{Re}\left(\frac{z h^{\prime}(z)}{Q(z)}\right)=$ $\operatorname{Re}\left(1+\frac{\xi}{\beta} q(z)+\frac{2 \mu}{\beta}(q(z))^{2}-\frac{z q^{\prime}(z)}{q(z)}+\frac{z q^{\prime \prime}(z)}{q^{\prime}(z)}\right)>0$.

We obtain $\alpha+\beta \frac{z p^{\prime}(z)}{p(z)}+\mu(p(z))^{2}+\xi p(z)=\alpha+\beta \delta\left[\frac{z\left(D_{z}^{-\lambda} \phi(a, c ; z)\right)^{\prime}}{D_{z}^{-\lambda} \phi(a, c ; z)}-1\right]+\mu\left[\frac{D_{z}^{-\lambda} \phi(a, c ; z)}{z}\right]^{2 \delta}+$ $\xi\left[\frac{D_{z}^{-\lambda} \phi(a, c ; z)}{z}\right]^{\delta}$

Using (8), we deduce $F_{p(U)}\left(\alpha+\beta \frac{z p^{\prime}(z)}{p(z)}+\mu(p(z))^{2}+\xi p(z)\right) \leq F_{q(U)}\left(\alpha+\beta q(z)+\mu(q(z))^{2}+\beta \frac{z q^{\prime}(z)}{q(z)}\right)$.

By an application of Lemma 1 we obtain $F_{p(U)} p(z) \leq F_{q(U)} q(z), z \in U$, i.e., $F_{D_{z}^{-\lambda} \phi(U)}\left(\frac{D_{z}^{-\lambda} \phi(a, c ; z)}{z}\right)^{\delta} \leq F_{q(U)} q(z), z \in U$ and $q$ is the fuzzy best dominant.

Corollary 1. Let $c$, a be complex numbers with $c \neq 0,-1,-2, \ldots$ and $\delta, \lambda>0$. Assume that (6) holds. If

$$
F_{\psi_{\lambda}^{a, c}(U)} \psi_{\lambda}^{a, c}(\delta, \alpha, \xi, \mu, \beta ; z) \leq F_{q(U)}\left(\alpha+\beta \frac{(A-B) z}{(1+A z)(1+B z)}+\mu\left(\frac{1+A z}{1+B z}\right)^{2}+\xi \frac{1+A z}{1+B z}\right)
$$

for $\xi, \alpha, \beta, \mu \in \mathbb{C}, \beta \neq 0,-1 \leq B<A \leq 1$, and $\psi_{\lambda}^{a, c}$ is introduced in (7), then

$$
F_{D_{z}^{-\lambda} \phi(U)}\left(\frac{D_{z}^{-\lambda} \phi(a, c ; z)}{z}\right)^{\delta} \leq F_{q(U)} \frac{1+A z}{1+B z}, \quad z \in U
$$

and $\frac{1+A z}{1+B z}$ is the fuzzy best dominant.

Proof. Consider in Theorem $1 q(z)=\frac{1+A z}{1+B z},-1 \leq B<A \leq 1$.

Corollary 2. Let $c$, a be complex numbers with $c \neq 0,-1,-2, \ldots$ and $\delta, \lambda>0$. Assume that (6) holds. If

$$
F_{\psi_{\lambda}^{a, c}(U)} \psi_{\lambda}^{a, c}(\delta, \alpha, \xi, \mu, \beta ; z) \leq F_{q(U)}\left(\alpha+\xi\left(\frac{1+z}{1-z}\right)^{\gamma}+\mu\left(\frac{1+z}{1-z}\right)^{2 \gamma}+\beta \frac{2 \gamma z}{1-z^{2}}\right),
$$

for $\xi, \alpha, \beta, \mu \in \mathbb{C}, \beta \neq 0,0<\gamma \leq 1$, where $\psi_{\lambda}^{a, c}$ is introduced in (7), then

$$
F_{D_{z}^{-\lambda} \phi(U)}\left(\frac{D_{z}^{-\lambda} \phi(a, c ; z)}{z}\right)^{\delta} \leq F_{q(U)}\left(\frac{1+z}{1-z}\right)^{\gamma}, z \in U
$$

and $\left(\frac{1+z}{1-z}\right)^{\gamma}$ is the fuzzy best dominant.

Proof. Theorem 1 give Corollary for $q(z)=\left(\frac{1+z}{1-z}\right)^{\gamma}, 0<\gamma \leq 1$.

Theorem 2. Let $q$ be analytic and univalent in $U$ such that $q(z) \neq 0$ and $\frac{z q^{\prime}(z)}{q(z)}$ be starlike univalent in $U$. Assume that

$$
\operatorname{Re}\left(\frac{\xi}{\beta} q(z)+\frac{2 \mu}{\beta}(q(z))^{2}\right)>0, \text { for } \mu, \xi, \beta \in \mathbb{C}, \beta \neq 0 .
$$


Let $c$, a be complex numbers with $c \neq 0,-1,-2, \ldots$ and $\delta, \lambda>0$. If $\psi_{\lambda}^{a, c}(\delta, \alpha, \xi, \mu, \beta ; z)$ is univalent in $U$ and $\left(\frac{D_{z}^{-\lambda} \phi(a, c ; z)}{z}\right)^{\delta} \in \mathcal{H}[0,(\lambda-1) \delta] \cap Q$, where $\psi_{\lambda}^{a, c}(\delta, \alpha, \xi, \mu, \beta ; z)$ is introduced in (7), then

$$
F_{q(U)}\left(\alpha+\beta \frac{z q^{\prime}(z)}{q(z)}+\mu(q(z))^{2}+\xi q(z)\right) \leq F_{\psi_{\lambda}^{a, c}(U)} \psi_{\lambda}^{a, c}(\delta, \alpha, \xi, \mu, \beta ; z)
$$

implies

$$
F_{q(U)} q(z) \leq F_{D_{z}^{-\lambda} \phi(U)}\left(\frac{D_{z}^{-\lambda} \phi(a, c ; z)}{z}\right)^{\delta}, z \in U,
$$

and $q$ is the fuzzy best subordinant.

Proof. Define $p(z):=\left(\frac{D_{z}^{-\lambda} \phi(a, c ; z)}{z}\right)^{\delta}, z \in U, z \neq 0$.

Consider $v(w):=\mu w^{2}+\xi w+\alpha$ and $\phi(w):=\frac{\beta}{w}$ it is evident that $v$ is analytic in $\mathbb{C}, \phi$ is analytic in $\mathbb{C} \backslash\{0\}$ and $\phi(w) \neq 0, w \in \mathbb{C} \backslash\{0\}$.

In this conditions $\frac{v^{\prime}(q(z))}{\phi(q(z))}=\frac{q^{\prime}(z)[\tilde{\xi}+2 \mu q(z)] q(z)}{\beta}$, which imply $\operatorname{Re}\left(\frac{v^{\prime}(q(z))}{\phi(q(z))}\right)=\operatorname{Re}\left(\frac{\tilde{\xi}}{\beta} q(z)+\frac{2 \mu}{\beta}(q(z))^{2}\right)>$ 0 , for $\xi, \beta, \mu \in \mathbb{C}, \beta \neq 0$.

We obtain

$$
F_{q(U)}\left(\alpha+\beta \frac{z q^{\prime}(z)}{q(z)}+\mu(q(z))^{2}+\xi q(z)\right) \leq F_{p(U)}\left(\alpha+\beta \frac{z p^{\prime}(z)}{p(z)}+\mu(p(z))^{2}+\xi p(z)\right) .
$$

Applying Lemma 2, we obtain

$$
F_{q(U)} q(z) \leq F_{D_{z}^{-\lambda} \phi(U)}\left(\frac{D_{z}^{-\lambda} \phi(a, c ; z)}{z}\right)^{\delta}, \quad z \in U,
$$

and $q$ is the fuzzy best subordinant.

Corollary 3. Let $c$, a be complex numbers with $c \neq 0,-1,-2, \ldots$ and $\delta, \lambda>0$. Assume that (10) holds. If $\left(\frac{D_{z}^{-\lambda} \phi(a, c ; z)}{z}\right)^{\delta} \in \mathcal{H}[0,(\lambda-1) \delta] \cap Q$ and

$$
F_{q(U)}\left(\alpha+\beta \frac{(A-B) z}{(1+A z)(1+B z)}+\mu\left(\frac{1+A z}{1+B z}\right)^{2}+\xi \frac{1+A z}{1+B z}\right) \leq F_{\psi_{\lambda}^{a, c}(U)} \psi_{\lambda}^{a, c}(\delta, \alpha, \xi, \mu, \beta ; z),
$$

for $\beta, \xi, \alpha, \mu \in \mathbb{C}, \beta \neq 0,-1 \leq B<A \leq 1$, where $\psi_{\lambda}^{a, c}$ is introduced in (7), then

$$
F_{q(U)}\left(\frac{1+A z}{1+B z}\right) \leq F_{D_{z}^{-\lambda} \phi(U)}\left(\frac{D_{z}^{-\lambda} \phi(a, c ; z)}{z}\right)^{\delta}, z \in U,
$$

and $\frac{1+A z}{1+B z}$ is the fuzzy best subordinant.

Proof. Theorem 2 for $q(z)=\frac{1+A z}{1+B z},-1 \leq B<A \leq 1$ give the corollary.

Corollary 4. Let $c$, a be complex numbers with $c \neq 0,-1,-2, \ldots$ and $\delta, \lambda>0$. Assume that (10) holds. If $\left(\frac{D_{z}^{-\lambda} \phi(a, c ; z)}{z}\right)^{\delta} \in \mathcal{H}[0,(\lambda-1) \delta] \cap Q$ and

$$
F_{q(U)}\left(\alpha+\beta \frac{2 \gamma z}{1-z^{2}}+\mu\left(\frac{1+z}{1-z}\right)^{2 \gamma}+\xi\left(\frac{1+z}{1-z}\right)^{\gamma}\right) \leq F_{\psi_{\lambda}^{a, c}(U)} \psi_{\lambda}^{a, c}(\delta, \alpha, \xi, \mu, \beta ; z),
$$


for $\beta, \xi, \alpha, \mu \in \mathbb{C}, 0<\gamma \leq 1, \beta \neq 0$, where $\psi_{\lambda}^{a, c}$ is introduced in (7), then

$$
F_{q(U)}\left(\frac{1+z}{1-z}\right)^{\gamma} \leq F_{D_{z}^{-\lambda} \phi(U)}\left(\frac{D_{z}^{-\lambda} \phi(a, c ; z)}{z}\right)^{\delta}, z \in U,
$$

and $\left(\frac{1+z}{1-z}\right)^{\gamma}$ is the fuzzy best subordinant.

Proof. Theorem 2 for $q(z)=\left(\frac{1+z}{1-z}\right)^{\gamma}, 0<\gamma \leq 1$, give the corollary.

Theorems 1 and 2 combined give the following sandwich theorem.

Theorem 3. Let $q_{1}$ and $q_{2}$ be analytic and univalent in $U$ such that $q_{1}(z) \neq 0$ and $q_{2}(z) \neq$ 0 , for all $z \in U$, with $\frac{z q_{1}^{\prime}(z)}{q_{1}(z)}$ and $\frac{z q_{2}^{\prime}(z)}{q_{2}(z)}$ being starlike univalent. Suppose that $q_{1}$ satisfies (6) and $q_{2}$ satisfies (10). Let $c$, a be complex numbers with $c \neq 0,-1,-2, \ldots$ and $\delta, \lambda>0$. If $\psi_{\lambda}^{a, c}(\delta, \alpha, \xi, \mu, \beta ; z)$ is as introduced in (7) univalent in $U$ and $\left(\frac{D_{z}^{-\lambda} \phi(a, c ; z)}{z}\right)^{\delta} \in \mathcal{H}[0,(\lambda-1) \delta] \cap$ $Q$, then

$$
\begin{gathered}
F_{q_{1}(U)}\left(\alpha+\beta \frac{z q_{1}^{\prime}(z)}{q_{1}(z)}+\mu\left(q_{1}(z)\right)^{2}+\xi q_{1}(z)\right) \leq F_{\psi_{\lambda}^{a, c}(U)} \psi_{\lambda}^{a, c}(\delta, \alpha, \xi, \mu, \beta ; z) \\
\leq F_{q_{2}(U)}\left(\alpha+\xi q_{2}(z)+\mu\left(q_{2}(z)\right)^{2}+\beta \frac{z q_{2}^{\prime}(z)}{q_{2}(z)}\right)
\end{gathered}
$$

for $\beta, \xi, \alpha, \mu \in \mathbb{C}, \beta \neq 0$, implies

$$
F_{q_{1}(U)} q_{1}(z) \leq F_{D_{z}^{-\lambda} \phi(U)}\left(\frac{D_{z}^{-\lambda} \phi(a, c ; z)}{z}\right)^{\delta} \leq F_{q_{2}(U)} q_{2}(z), \quad z \in U,
$$

and $q_{1}$ and $q_{2}$ are respectively the fuzzy best subordinant and the fuzzy best dominant.

For $q_{1}(z)=\frac{1+A_{1} z}{1+B_{1} z}, q_{2}(z)=\frac{1+A_{2} z}{1+B_{2} z}$, where $-1 \leq B_{2}<B_{1}<A_{1}<A_{2} \leq 1$, we obtain the following corollary.

Corollary 5. Let $c$, a be complex numbers with $c \neq 0,-1,-2, \ldots$ and $\delta, \lambda>0$. Assume that (6) and (10) hold. If $\left(\frac{D_{z}^{-\lambda} \phi(a, c ; z)}{z}\right)^{\delta} \in \mathcal{H}[0,(\lambda-1) \delta] \cap Q$ and

$$
\begin{array}{r}
F_{q_{1}(U)}\left(\alpha+\beta \frac{\left(A_{1}-B_{1}\right) z}{\left(1+A_{1} z\right)\left(1+B_{1} z\right)}+\mu\left(\frac{1+A_{1} z}{1+B_{1} z}\right)^{2}+\xi \frac{1+A_{1} z}{1+B_{1} z}\right) \leq F_{\psi_{\lambda}^{a, c}(U)} \psi_{\lambda}^{a, c}(\delta, \alpha, \xi, \mu, \beta ; z) \\
\leq F_{q_{2}(U)}\left(\alpha+\beta \frac{\left(A_{2}-B_{2}\right) z}{\left(1+A_{2} z\right)\left(1+B_{2} z\right)}+\mu\left(\frac{1+A_{2} z}{1+B_{2} z}\right)^{2}+\xi \frac{1+A_{2} z}{1+B_{2} z}\right)
\end{array}
$$

for $\beta, \xi \alpha, \mu, \in \mathbb{C}, \beta \neq 0,-1 \leq B_{2} \leq B_{1}<A_{1} \leq A_{2} \leq 1$, where $\psi_{\lambda}^{a, c}$ is introduced in (7), then

$$
F_{q_{1}(U)}\left(\frac{1+A_{1} z}{1+B_{1} z}\right) \leq F_{D_{z}^{-\lambda} \phi}\left(\frac{D_{z}^{-\lambda} \phi(a, c ; z)}{z}\right)^{\delta} \leq F_{q_{2}(U)} \frac{1+A_{2} z}{1+B_{2} z},
$$

hence $\frac{1+A_{1} z}{1+B_{1} z}$ and $\frac{1+A_{2} z}{1+B_{2} z}$ are the fuzzy best subordinant and the fuzzy best dominant, respectively. 
Corollary 6. Let $c$, a be complex numbers with $c \neq 0,-1,-2, \ldots$ and $\delta, \lambda>0$. Assume that (6) and (10) hold. If $\left(\frac{D_{z}^{-\lambda} \phi(a, c ; z)}{z}\right)^{\delta} \in \mathcal{H}[0,(\lambda-1) \delta] \cap Q$ and

$$
\begin{gathered}
F_{q_{1}(U)}\left(\alpha+\beta \frac{2 \gamma_{1} z}{1-z^{2}}+\mu\left(\frac{1+z}{1-z}\right)^{2 \gamma_{1}}+\xi\left(\frac{1+z}{1-z}\right)^{\gamma_{1}}\right) \leq F_{\psi_{\lambda}^{a, c}(U)} \psi_{\lambda}^{a, c}(\delta, \alpha, \xi, \mu, \beta ; z) \\
\leq F_{q_{2}(U)}\left(\alpha+\xi\left(\frac{1+z}{1-z}\right)^{\gamma_{2}}+\mu\left(\frac{1+z}{1-z}\right)^{2 \gamma_{2}}+\beta \frac{2 \gamma_{2} z}{1-z^{2}}\right),
\end{gathered}
$$

for $\beta, \xi \alpha, \mu, \in \mathbb{C}, \beta \neq 0,0<\gamma_{1}, \gamma_{2} \leq 1$, where $\psi_{\lambda}^{a, c}$ is introduced in (7), then

$$
F_{q_{1}(U)}\left(\frac{1+z}{1-z}\right)^{\gamma_{1}} \leq F_{D_{z}^{-\lambda} \phi(U)}\left(\frac{D_{z}^{-\lambda} \phi(a, c ; z)}{z}\right)^{\delta} \leq F_{q_{2}(U)}\left(\frac{1+z}{1-z}\right)^{\gamma_{2}}
$$

hence $\left(\frac{1+z}{1-z}\right)^{\gamma_{1}}$ and $\left(\frac{1+z}{1-z}\right)^{\gamma_{2}}$ are the fuzzy best subordinant and the fuzzy best dominant, respectively.

\section{Conclusions}

The interesting operator presented in Definition 3 was previously defined and studied related to several aspects of differential subordination theory in [36] as a fractional integral of confluent hypergeometric function. In this paper, the study of the operator is continued using the recently introduced notions of fuzzy differential subordination and fuzzy differential superordination as a result of the preoccupation with adapting the classical notions of differential subordination and superordination to fuzzy sets theory. Fuzzy differential subordinations and fuzzy differential superordinations are presented in the original theorems giving their best fuzzy dominant and best fuzzy subordinant, respectively. Using particular functions, interesting corollaries are presented that could inspire future studies related to the univalence of the operator. A sandwich-type result is obtained in the last theorem combining the results proved using the two theories of fuzzy differential subordination and fuzzy differential superordination. Since the operator gives nice results in studies done with both theories, it could be used for introducing new fuzzy classes of analytic functions and performing studies on those classes using both theories.

Finding applications in other domains for the operator and for the results of the fuzzy investigation presented in this paper remains an open problem to which future interdisciplinary applications are desired.

Funding: This research received no external funding.

Institutional Review Board Statement: Not applicable.

Informed Consent Statement: Not applicable.

Data Availability Statement: Not applicable.

Conflicts of Interest: The author declares no conflict of interest.

\section{References}

1. Zadeh, L.A. Fuzzy Sets. Inf. Control 1965, 8, 338-353. [CrossRef]

2. Dzitac, I.; Filip, F.G.; Manolescu, M.J. Fuzzy Logic Is Not Fuzzy: World-renowned Computer Scientist Lotfi A. Zadeh. Int. J. Comput. Commun. Control. 2017, 12, 748-789. [CrossRef]

3. Dzitac, S.; Nădăban, S. Soft Computing for Decision-Making in Fuzzy Environments: A Tribute to Professor Ioan Dzitac. Mathematics 2021, 9, 1701. [CrossRef]

4. Oros, G.I.; Oros, G. The notion of subordination in fuzzy sets theory. Gen. Math. 2011, 19, 97-103.

5. Miller, S.S.; Mocanu, P.T. Second order-differential inequalities in the complex plane. J. Math. Anal. Appl. 1978, 65, 298-305. [CrossRef]

6. Miller, S.S.; Mocanu, P.T. Differential subordinations and univalent functions. Michig. Math. J. 1981, 28, 157-171. [CrossRef] 
7. Oros, G.I.; Oros, G. Fuzzy differential subordination. Acta Univ. Apulensis 2012, 3, 55-64.

8. Oros, G.I.; Oros, G. Dominants and best dominants in fuzzy differential subordinations. Stud. Univ. Babeş-Bolyai Math. 2012, 57, 239-248.

9. Oros, G.I.; Oros, G. Briot-Bouquet fuzzy differential subordination. An. Univ. Oradea Fasc. Mat. 2012, $19,83-87$.

10. Miller, S.S.; Mocanu, P.T. Differential Subordinations. Theory and Applications; Marcel Dekker, Inc.: New York, NY, USA; Basel, Switzerland, 2000.

11. Alb Lupaş, A. On special fuzzy differential subordinations using convolution product of Sălăgean operator and Ruscheweyh derivative. J. Comput. Anal. Appl. 2013, 15, 1484-1489.

12. Venter, A.O. On special fuzzy differential subordination using Ruscheweyh operator. An. Univ. Oradea Fasc. Mat. 2015, XXII, 167-176.

13. Alb Lupaş, A. A note on special fuzzy differential subordinations using multiplier transformation. An. Univ. Oradea Fasc. Mat. 2016, XXIII, 183-191.

14. Wanas, A.K.; Majeed, A.H. Fuzzy differential subordinations for prestarlike functions of complex order and some applications. Far East J. Math. Sci. 2017, 102, 1777-1788. [CrossRef]

15. Atshan, W.G.; Hussain, K.O. Fuzzy Differential Superordination. Theory Appl. Math. Comput. Sci. 2017, 7, 27-38.

16. Ibrahim, R.W. On the subordination and superordination concepts with applications. J. Comput. Theor. Nanosci. 2017, 14, 2248-2254. [CrossRef]

17. Thilagavathi, K. Fuzzy subordination and superordination results for certain subclasses of analytic functions associated with Srivastava-Attitya operator. Int. J. Pure Appl. Math. 2018, 118, 921-929.

18. Oros, G.I. New fuzzy differential subordinations. Commun. Fac. Sci. Univ. Ank. Ser. A1 Math. Stat. 2021, 70, 229-240.

19. El-Deeb, S.M.; Alb Lupaş, A. Fuzzy differential subordinations associated with an integral operator. An. Univ. Oradea Fasc. Mat. 2020, XXVII, 133-140.

20. El-Deeb, S.M.; Oros, G.I. Fuzzy differential subordinations connected with the linear operator. Math. Bohem. 2021, 1-10.

21. Alb Lupaş, A.; Oros, G.I. New Applications of Sălăgean and Ruscheweyh Operators for Obtaining Fuzzy Differential Subordinations. Mathematics 2021, 9, 2000. [CrossRef]

22. Srivastava, H.M.; El-Deeb, S.M. Fuzzy Differential Subordinations Based upon the Mittag-Leffler Type Borel Distribution. Symmetry 2021, 13, 1023. [CrossRef]

23. Wanas, A.K.; Majeed, A.H. Fuzzy subordination results for fractional integral associated with generalized Mittag-Leffler function. Eng. Math. Lett. 2019, 2019, 10.

24. Rashid, S.; Ashraf, R.; Akdemir, A.O.; Alqudah, M.A.; Abdeljawad, T.; Mohamed, M.S. Analytic Fuzzy Formulation of a Time-Fractional Fornberg-Whitham Model with Power and Mittag-Leffler Kernels. Fractal Fract. 2021, 5, 113. [CrossRef]

25. Oros, G.I. Fuzzy Differential Subordinations Obtained Using a Hypergeometric Integral Operator. Mathematics 2021, 9, 2539. [CrossRef]

26. Rashid, S.; Khalid, A.; Sultana, S.; Hammouch, Z.; Shah, R.; Alsharif, A.M. A novel analytical view of time-fractional Korteweg-De Vries equations via a new integral transform. Symmetry 2021, 13, 1254. [CrossRef]

27. Baleanu, D.; Jajarmi, A.; Mohammadi, H.; Rezapour, S. A new study on the mathematical modelling of human liver with Caputo-Fabrizio fractional derivative. Chaos Solitons Fract. 2020, 134, 109705. [CrossRef]

28. Srivastava, H.M.; Jan, R.; Jan, A.; Deebai, W.; Shutaywi, M. Fractional-calculus analysis of the transmission dynamics of the dengue infection. Chaos 2021, 31, 53130. [CrossRef]

29. Srivastava, H.M.; Bansal, M.; Harjule, P. A study of fractional integral operators involving a certain generalized multi-index Mittag-Leffler function. Math. Methods Appl. Sci. 2018, 41, 6108-6121. [CrossRef]

30. Saxena, R.K.; Purohit, S.D.; Kumar D. Integral Inequalities Associated with Gauss Hypergeometric Function Fractional Integral Operators. Proc. Natl. Acad. Sci. India Sect. A Phys. Sci. 2018, 88, 27-31. [CrossRef]

31. Rashid, S.; Khalid, A.; Bazighifan, O.; Oros, G.I. New modifications of integral inequalities via $\gamma$-convexity pertaining to fractional calculus and their applications. Mathematics 2021, 9, 1753. [CrossRef]

32. Alb Lupaş, A. Properties on a subclass of analytic functions defined by a fractional integral operator. J. Comput. Anal. Appl. 2019, $27,506-510$.

33. Alb Lupaş, A. Inequalities for Analytic Functions Deffined by a Fractional Integral Operator. In Frontiers in Functional Equations and Analytic Inequalities; Anastassiou, G., Rassias, J., Eds.; Springer: Berlin, Germany, 2020; pp. 731-745.

34. Cho, N.E.; Aouf, M.K.; Srivastava, R. The principle of differential subordination and its application to analytic and $p$-valent functions defined by a generalized fractional differintegral operator. Symmetry 2019, 11, 1083. [CrossRef]

35. Ghanim, F.; Al-Janaby, H.F. An analytical study on Mittag-Lefler-confluent hypergeometric functions with fractional integral operator. Math. Methods Appl. Sci. 2020, 44, 3605-3614. [CrossRef]

36. Alb Lupaş, A.; Oros, G.I. Differential Subordination and Superordination Results Using Fractional Integral of Confluent Hypergeometric Function. Symmetry 2021, 13, 327. [CrossRef]

37. Srivastava, H.M.; Owa, S. An application of the fractional derivative. Mud Jpn. 1984, 29, 383-389.

38. Gal, S.G.; Ban, A.I. Elemente de Matematică Fuzzy; University of Oradea: Oradea, Romania, 1996. 